\title{
Assessment of the Effect of Dielectrophoresis (DEP) on the Viability of Activated Sludge Biomass
}

\author{
B. Larbi, A. Ltaief, A. Hawari, F. Du, M. Baune, and J. Thöming
}

\begin{abstract}
Effective application of dielectrophoretic forces in membrane bioreactor (MBR) systems has the potential to suppress membrane fouling. Nevertheless, minimizing the impact of the electric field on biomass suspension is essential to maintain the efficiency of the wastewater treatment process. In this study, interdigitated cylindrical electrodes (IDE), placed inside the membrane module, were used to generate dielectrophoresis (DEP) in an MBR system. The viability of the biomass was determined at different voltages $(5-150 \mathrm{~V})$ after 1-hour exposure to a pulsed alternating current (AC) electric field. The results of the behavioral response of the microorganisms revealed that at low voltage applications, the bacteria exhibited good performance and no major impact was found on their viability/metabolism. Whereas at high voltage applications (beyond $100 \mathrm{~V}$ ), the current intensity and medium temperature increased due to the joule heating effect and caused a significant decline in the bacterial activity and pollutant removal efficiency as a result of bacterial lysis.
\end{abstract}

Index Terms-Bacterial lysis, dielectrophoresis, interdigitated cylindrical electrodes, microorganisms, membrane bioreactor.

\section{INTRODUCTION}

Membrane Bioreactors (MBRs) have recently gained wide utilization in the treatment of wastewater. They are well known for their ease of operation and enhanced effluent quality. Membrane fouling remains a significant issue in MBRs that reduces the filtration performance and increases the operational costs, as it becomes more necessary to clean or replace the fouled membranes. Many methods have been proposed to alleviate this issue among which the application of an electric field using electrophoresis (EP). It was demonstrated that the applied electric field of high voltages inside the bioreactor has the potential to repel particles away from the membrane [1]-[3]. Yet, it has been reported that different voltage strengths will have different effects on the microorganisms [4]-[7].

Many studies have focused on the use of electrophoresis, this is usually associated with the assumption that the bioparticles are negatively charged, however the feed

Manuscript received June 9, 2017; revised September 12, 2017. This publication was made possible by NPRP grant \# (NPRP7-089-2-044) from the Qatar National Research Fund (a member of The Qatar Foundation). The statements made herein are solely the responsibility of the authors.

B. Larbi, A. Ltaief, and A. Hawari are with the Department of Civil and Architectural Engineering, College of Engineering, Qatar University, 2713 Doha, Qatar (e-mail: bouthaina.larbi@qu.edu.qa, amina.ltaeif@qu.edu.qa, a.hawari@qu.edu.qa).

F. Du, M. Baune, and J. Thöming are with Center for Environmental Research and Sustainable Technology, University of Bremen, Leobener Str., D 28359 Bremen, Germany (e-mail: fei.du@uni-bremen.de, m.baune@uni-bremen.de, thoming@uni-bremen.de). suspension complexity inhibits the use of such an assumption [8], [9]. In addition, direct current (DC) electric field is often used to generate EP which induces higher energy consumption. Recently, Dielectrophoresis (DEP) has been applied successfully in a MBR to control fouling. This technique involves inducing a non-uniform electric field that creates a dielectric polarization on the particles and causes their translational motion [10]. DEP has gained wide utilization in many nanoscale biological applications. In the wastewater industry, it was used by Du et al. and Hawari et al., [9], [11] to suppress fouling in membrane treatment processes. Upon the application of dielectrophoretic forces to wastewater, a net repulsive force is created that causes the migration of particles away from the membrane as a result of the differences in permittivity between the medium and bioparticles. In their study, Du et al., successfully increased the permeate flux by $50 \%$. Then, they further enhanced the permeate flux by $68 \%$ [12]. The same research group [11] demonstrated later that a stronger electric field induces stronger DEP forces on the particles that alleviate fouling more effectively and result in a better permeate flux. On the other hand, they found that stronger electric fields are associated with joule heating. This effect creates temperature gradients that introduce buoyancy forces and affect the particle's motion which may reduce the fouling suppression effectiveness in specific conditions.

Yet the influence of such a process on the biomass viability has not been studied. Therefore, the aim of this research is to study the effect of pulsed DEP electric field and joule heating on microorganisms of the activated sludge. The dielectrophoretic forces will be generated by interdigitated electrodes placed below the membrane, through which a low frequency $(50 \mathrm{~Hz})$ alternating current (AC) will be applied intermittently at different voltages ranging from $(5$, $25,50,100$, and $150 \mathrm{~V})$. Bacteria viability will be determined through quantitative analysis on its physiological changes.

\section{MATERIALS AND METHOD}

\section{A. MBR System}

A $4 \mathrm{~L}$ bioreactor was used for this study, into which a Chlorinated Polyethylene (CPE) membrane module was submerged (Fig. 1a). An array of interdigitated electrodes was integrated inside the membrane module as shown in Fig. 1b. The electrodes were coated with a thin titanium dioxide layer to insulate them from electrochemical corrosion on their surface and to protect from the risk of human electric shock. The membrane module was kept submerged throughout the experiment by controlling the water level. And a magnetic stirrer was used to ensure complete 
homogeneity in the bioreactor. Activated sludge from a local wastewater treatment plant was used within 48 hrs. of its extraction and was supplied continuously with appropriate aeration. The medium temperature, $\mathrm{pH}$, and conductivity were monitored throughout the experiment using WTW Multi3430 multiparameter meter.

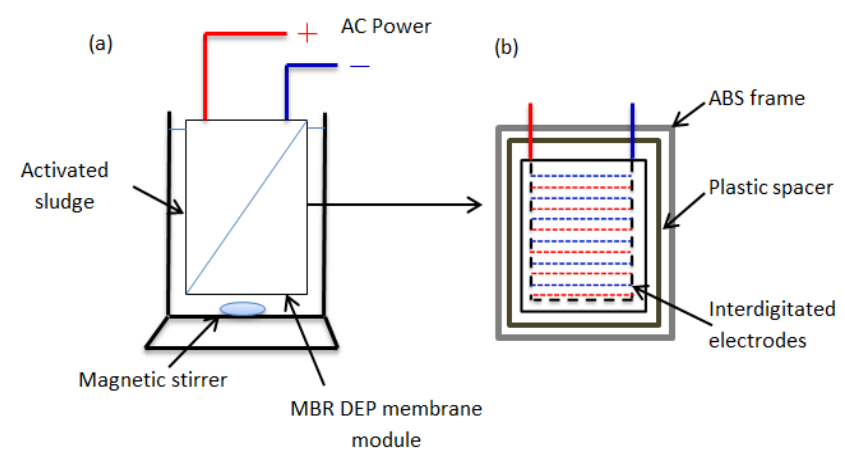

Fig. 1. Schematic diagram for the MBR system (a), cross sectional diagram for the membrane module (b).

\section{B. Methodology}

The membrane module was connected to an AC power supply. The applied voltages were set to 5, 25, 50, 100, and $150 \mathrm{~V}$ at $50 \mathrm{~Hz}$, and applied intermittently for 1 hour; with 10 $\mathrm{s}$ of electric field on and $15 \mathrm{~s}$ of the electric field off.

The physiological changes of the sludge upon DEP application at different voltages was determined through analyzing for the total organic carbon (TOC), Ammonium concentration $\left[\mathrm{NH}_{4}{ }^{+}\right]$and oxygen uptake rate (OUR).

The TOC was obtained by filtering the withdrawn sample through a $1.5 \mu \mathrm{m}$ glass microfiber filter, and the filtrate was used to determine the TOC using the Aurora 1030W TOC Analyser.

The Ammonium concentration was obtained in duplicates using the Nesslerization method: Anhydrous Ammonium Chloride solution was initially used to calibrate the Orion AquaMate UV-VIS Spectrophotometer at a wavelength of $425 \mathrm{~nm}$, then $\left[\mathrm{NH}_{4}{ }^{+}\right]$of the samples was obtained after Nesslerization of the filtrate.

To obtain the oxygen uptake rate, the following standard method was applied [13] a $200 \mathrm{ml}$ of the biological suspension was withdrawn from the MBR at the end of each voltage application in which a Dissolved oxygen (DO) probe is inserted. The sample was stirred continuously to avoid sludge settling and tightly sealed to isolate the contents from the external oxygen. DO concentrations were recorded over a 10 minute period. The slope of DO-time represents the oxygen uptake rate in $\mathrm{mg} \mathrm{O}_{2} /$ L.min.

\section{RESUlTS AND DisCUSSION}

\section{A. Effect of DEP on the Medium Parameters}

The temperature change and the electric current inside the bioreactor were monitored during the course of the experiments and are displayed in Fig. 2a and b, respectively. At low voltage applications of $5 \mathrm{~V}, 25 \mathrm{~V}$ and $50 \mathrm{~V}$, the temperature change was almost negligible $\left( \pm 2{ }^{\circ} \mathrm{C}\right)$. However, the temperature was found to increase significantly at high voltage applications to values beyond the optimum temperature $\left(31^{\circ} \mathrm{C}\right)$ for the biological reactions of the studied mesophilic microorganisms [14]. At $100 \mathrm{~V}$ the temperature rose by $47 \%$ reaching $41.3^{\circ} \mathrm{C}$ at the end of the test. At $150 \mathrm{~V}$ the temperature rose by $123 \%$ reaching $67.4^{\circ} \mathrm{C}$ after 50 minutes of pulsed DEP application after which the test had to be stopped for safety.

Similarly, the electric current was affected by the same degree; at low voltage applications, the current increase was insignificant whereas at $100 \mathrm{~V}$ and $150 \mathrm{~V}$ the electric current reached $2.33 \mathrm{~A}$ and $6 \mathrm{~A}$ respectively at the end of the test. This sudden change in temperature and current intensity at high voltage applications are speculated to be caused by the electrothermal effect as a result of joule heating. The highly conductive medium, wastewater in this case, along with the high electric field mutually caused the increase in the electric current. Consequently, producing heat that spread through the medium. On the other hand, the measured conductivity and the $\mathrm{pH}$ of the medium were invariant throughout the tests.

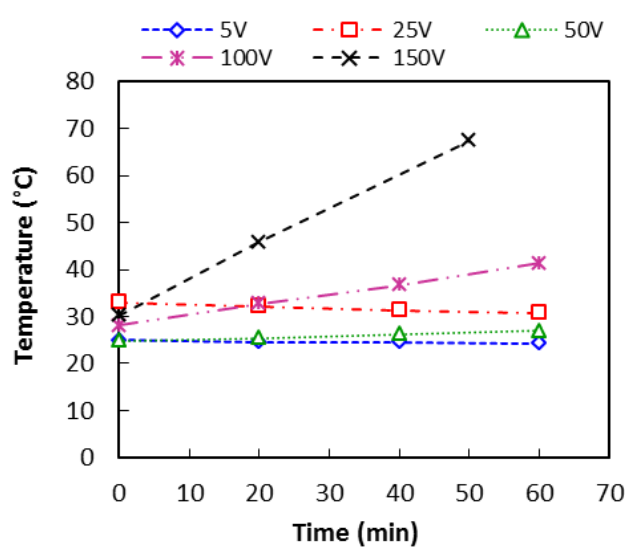

(a)

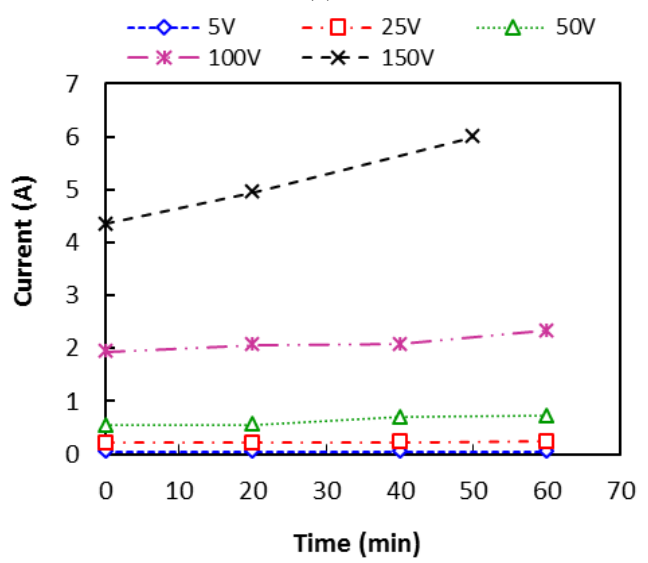

(b)

Fig. 2. Temperature (a) and electric current (b) change inside the MBR.

\section{B. Effect of DEP on the Medium Parameters}

According to the experimental results in Fig. 3a, one can conclude that successful TOC removal after 1 hour of pulsed DEP induction was obtained at low voltages. For instance, the TOC was removed by $39.1 \%$ when $25 \mathrm{~V}$ of pulsed DEP was applied. Similarly, Ammonium removal by the nitrifying bacteria has also been successful at these low voltages with high efficiency. Fig. $3 b$ shows the decline in ammonium concentration at the end of the test for the $25 \mathrm{~V}$ and $50 \mathrm{~V}$. These results explain that at low voltage applications the bacterial activity is enhanced 
through an increase in substrate metabolism. Indeed, these results agree with many studies conducted at low voltages using electrophoretic forces [6], [7].

Yet, at high voltage applications of $100 \mathrm{~V}$ the TOC removal decreased to $20.2 \%$. More importantly, after 50 minutes of $150 \mathrm{~V}$ application TOC concentration increased by $121.9 \%$. This increase in TOC at high voltage applications suggests an increase in the soluble microbial products (SMP). These are intracellular organic components that get released out from the microorganisms as a result of cell wall perforations. In addition, the increase in TOC can also be explained by an increase in the extracellular polymeric substances (EPS) namely polysaccharides and proteins; the EPS are believed to act as the glue that binds cells together and result in their bio-flocculation [2], [3].

Likewise, the ammonium concentration in the bulk solution has obviously increased rapidly at high voltage applications which can be due to the denaturation of the proteins releasing more ammonium ions into the bulk.

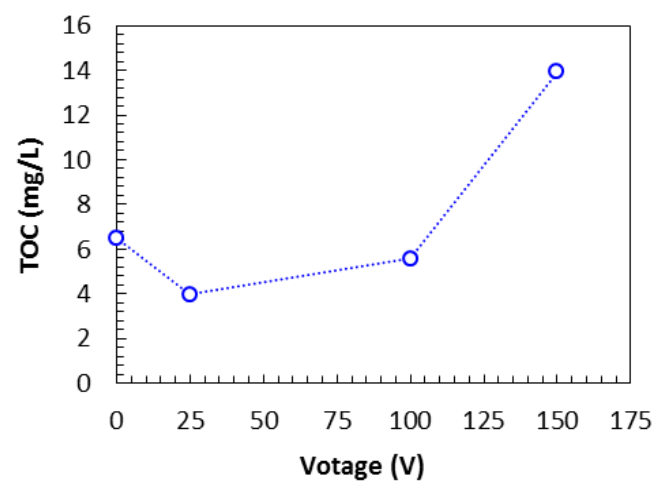

(a)

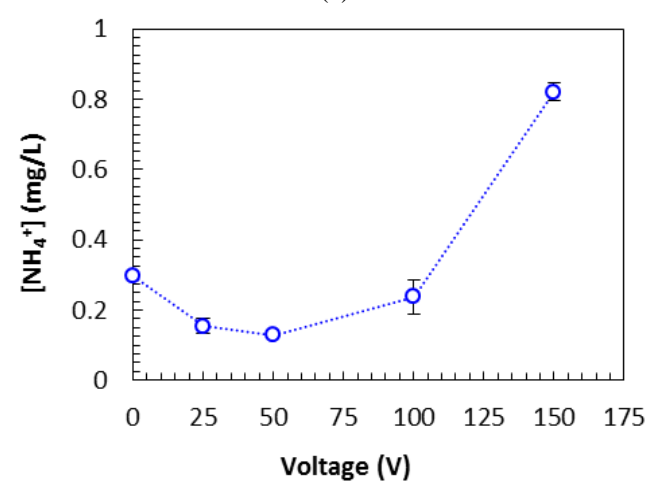

(b)

Fig. 3. TOC (a) and Ammonium concentration (b) after 60 minutes of different voltage strength applications.

With the application of high electric fields in a highly conductive medium, the joule heating effect is inevitable and caused the electric currents to increase above $2 \mathrm{~A}$. The high current intensity could have potentially caused bacterial lysis and stimulated the production of SMP, EPS, and ammonium in the bulk. Previous study on the effect of DC-electrophoretic forces [4] also found that above a current intensity of 2 A the bacteria destruction rate increases, due to the formation of nanoscale defects at the membrane wall causing the cytoplasmic contents to escape. The joule heating effect has also inhibited the metabolism of the mesophilic microorganisms including the nitrifying bacteria by the rise in fluid temperature to values beyond $39{ }^{\circ} \mathrm{C}$ [14] that caused protein denaturation.
The microbial community inside the bioreactor was assessed by determining the oxygen uptake rate at the end of the experiment for each voltage application as shown in Fig. 4. Remarkably, the OUR trend decreases with the voltage strength. For instance, at the end of the test, the OUR dropped by $28 \%$ for the $25 \mathrm{~V}$ application whereas it dropped by $79.7 \%$ when $150 \mathrm{~V}$ is supplied. The significant reduction in OUR at high voltage applications further explain the reduction in microbial activity of the cells in the medium as a result of bacterial lysis.

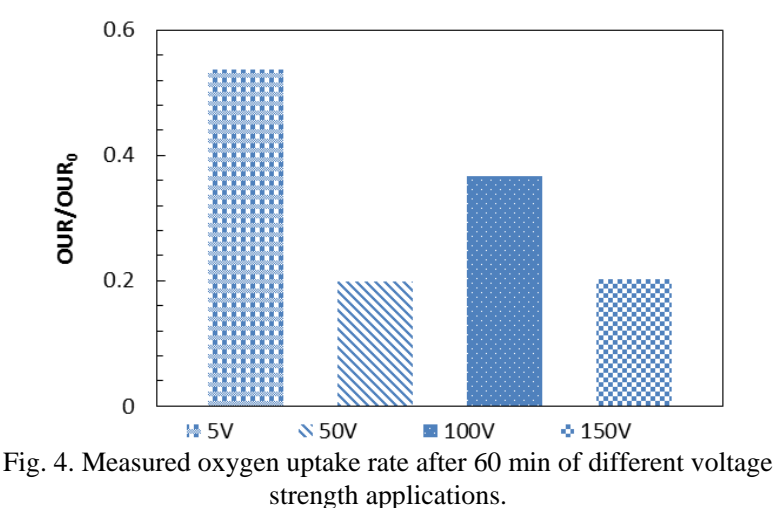

\section{CONCLUSION}

Despite the effectiveness of strong electric fields at generating high DEP forces to suppress membrane fouling and enhance the permeate flux, it was demonstrated from this study that high voltage applications are associated with the joule heating effect. This caused the generation of high current intensities that in return increased the medium temperature to values beyond the optimal temperature for the studied bacterial viability, eventually resulting in cell wall destruction and bacterial lysis. More research work on bacteria viability will be performed in future studies through extending the DEP force duration and measuring additional parameters for verification. This will, in expectation, enable us to determine the optimum working conditions for the DEP MBR system to control fouling while preserving the microbial community.

\section{ACKNOWLEDGMENT}

This publication was made possible by NPRP grant \# (NPRP7-089-2-044) from the Qatar National Research Fund (a member of The Qatar Foundation). The statements made herein are solely the responsibility of the authors. Also the authors would like to thank the public works authority of Qatar (ASHGAL) for their supply of the activated sludge samples.

\section{REFERENCES}

[1] J. Y. Kim, J. H. Lee, I. S. Chang, and C. W. Yi, "High voltage impulse electric fields: Disinfection kinetics and its effect on membrane bio-fouling," Desalination, vol. 283, pp. 111-116, 2011.

[2] J. S. Lee and I. S. Chang, "Membrane fouling control and sludge solubilization using high voltage impulse (HVI) electric fields," Process Biochemistry, vol. 49, pp. 858-862, 2014.

[3] J. Zhang, A. Satti, X. Chen, K. Xiao, J. Sun, X. Yan, P. Liang, X. Zhang, and X. Huang, "Low-voltage electric field applied into MBR for fouling suppression: Performance and mechanisms," Chemical Engineering Journal, vol. 273, pp. 223-230, 2015. 
[4] N. Boudjema, N. Drouiche, N. Abdi, H. Grib, H. Lounici, A. Pauss, and N. Mameri, "Treatment of Oued El Harrach river water by electrocoagulation noting the effect of the electric field on microorganisms," Journal of the Taiwan Institute of Chemical Engineers, vol. 45, pp. 1564-1570, 2014.

[5] M. Loeffler, W. Schmidt, R. Schuhmann, and A. Röttering, "Treatment of Sewage Sludge with Pulsed Electric Fields Principal effects of pulsed electrical fields on rubber foils under water Experimental Set-up," presented at International Conference on Pulsed Power Applications, 2001.

[6] V. Wei, M. Elektorowicz, and J. A. Oleszkiewicz, "Influence of electric current on bacterial viability in wastewater treatment," Water Research, vol. 45, pp. 5058-62, 2011.

[7] W. Huang, W. Wang, W. Shi, Z. Lei, Z. Zhang, R. Chen, and B. Zhou, "Use low direct current electric field to augment nitrification and structural stability of aerobic granular sludge when treating low COD/NH4-N wastewater," Bioresource Technology, vol. 171, pp. 139-44, 2014.

[8] H. M. Kyllonen, P. Pirkonen, and M. Nystrom, "Membrane filtration enhanced by ultrasound: a review," Desalination, vol. 181, pp. 319-335, 2005.

[9] F. Du, A. Hawari, M. Baune, and J. Thöming, "Dielectrophoretically intensified cross-flow membrane filtration," Journal of Membrane Science, vol. 336, pp. 71-78, 2009.

[10] H. A. Pohl, K. Pollock, and J. S. Crane, "Dielectrophoretic force: A comparison of theory and experiment," Journal of Biological Physics, vol. 6, pp. 133-160, 1978.

[11] A. Hawari, F. Du, M. Baune, and J. Thöming, "A fouling suppression system in submerged membrane bioreactors using dielectrophoretic forces," Journal of Environmental Sciences, vol. 29, pp. 139-145, 2015.

[12] F. Du, P. Ciaciuch, S. Bohlen, Y. Wang, M. Baune, and J. Thöming, "Intensification of cross-flow membrane filtration using dielectrophoresis with a novel electrode configuration," Journal of Membrane Science, vol. 448, pp. 256-261, 2013.

[13] E. W. Rice, R. B. Baird, A. D. Eaton, and L. S. Clesceri, Standard Methods for the Examination of Water and Wastewater, American Public Health Association, American Water Works Association, Water Environment Federation, 2012.

[14] W. W. Eckenfelder, D. L Ford, and A. J. Englande, Industrial Water Quality, 4th Edition: The McGraw-Hill Companies, 2009.

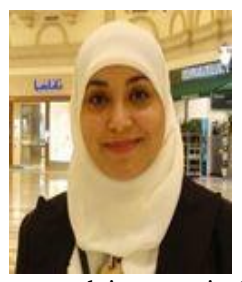

Bouthaina Larbi received her Ph.D. degree in civil engineering from Paris-Est University, Paris, France, in 2014. She works currently as a postdoctoral research fellow in the Department of Civil and Architectural Engineering at Qatar University, Qatar. Her most important publications concern mass transport in cement based materials and membrane fouling suppression using dielectrophoresis. Her research interests include mass transport in porous media, membrane fouling and wastewater treatment process.

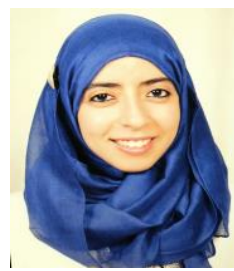

Amina Ltaief achieved her master of engineering degree in chemical engineering from Imperial College London University in 2015. She worked in Shell Technology Center in Amsterdam, Netherlands as a summer intern in the Research and Development Department in 2014 and in GlaxoSmithKline in the $\mathrm{UK}$ as a process engineer trainee. She is now working as a research assistant in the Department of Research and Graduate Studies at Qatar University in Qatar, on topics related to wastewater treatment.

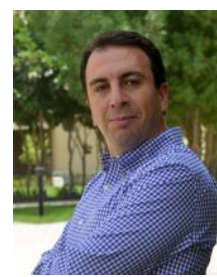

Alaa H. Hawari obtained his Ph.D. degree in environmental engineering at Concordia University, Montreal, Canada in 2004. Currently, he is associate professor in the Department of Civil and Architectural Engineering at Qatar University, Qatar. He has published many articles about membrane fouling, biosorption and selective adsorption. His research interests include water and wastewater treatment and water and sewer networks optimization.

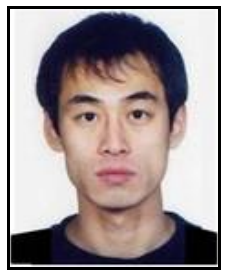

Fei Du obtained his Ph.D. at Bremen University, Bremen, Germany, in 2010. He is currently a senior researcher at Center for Environmental Research and Sustainable Technology, Chemical Engineering Recovery and Recycling. His research interests include separation of Micro and Nano particles, dielectrophoresis in porous media, dielectrophoresis dielectrophoresis. for gold separation and fouling suppression using

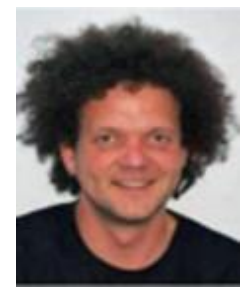

Michael Baune received his Ph.D. degree from Bremen University, Bremen, Germany in 2002. He is currently a Bremen Senior researcher. He is also a research fellow at the Center for Environmental Research and Sustainable Technology Chemica Engineering - Recovery and Recycling since 2002 His research interest include dielectrophoretic effects in porous structures, cellulose-based porous materials.

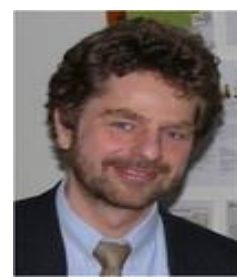

Jorg Thoming received his Ph.D. degree from the Technical University of Hamburg-Harburg (TUHH) Germany, in 1998. He is currently the director of the Center for Environmental Research and Sustainable Technology (UFT) and Professor at Bremen University. His research interests include mass transport and reaction processes in porous structures, dielectrophoresis effects, microbial fuel cells, membrane sciences, catalytic reactions. 